\title{
MONTE CARLO CALCULATION WITH UNQUENCHED WILSON FERMIONS
}

\author{
I. MONTVAY ${ }^{1}$ \\ II. Institut für Theoretische Physik, Universität Hamburg, Hamburg, Fed. Rep. Germany
}

Received 11 January 1984

\begin{abstract}
A Monte Carlo updating procedure taking into account the virtual quark loops is described. It is based on high-order hopping parameter expansion of the quark determinant for Wilson fermions. In a first test run Wilson loop expectation values are measured on a $6^{4}$ lattice at $\beta=5.70$ using 16 th order hopping parameter expansion for the quark determinant.
\end{abstract}

The inclusion of the effects of virtual fermion loops in Monte Carlo simulations of lattice gauge theories is an important and challenging problem. At present it is to a large extent unknown how much the Monte Carlo results obtained in the pure colour gauge sector or in the "quenched approximation" will be changed once light dynamical quarks are properly included in the computations of lattice quantum chromodynamics. There is a class of problems, like the screening of (fundamental) colour charge or the fragmentation of fast quarks into hadrons etc., which cannot even be formulated without virtual quark loops.

From the computational point of view the inclusion of dynamical fermions is, however, rather difficult because of the long range interaction induced by the virtual light fermion loops. Several methods proposed recently, like the pseudo-fermion method [1-3], stochastic method $[4,5]$ or the microcanonical method [6] are promising, but it is usually difficult to control their accuracy due to some approximations which affect the results only rather indirectly. In a recently proposed method based on high order numerical hopping parameter expansion $[7,8]$ the committed errors are easier to control. The fermion determinant is, however, not included in the updating but it is treated essentially as a part of the expectation value. In the case of light fermions this is the source of large fluctuations prohibiting the collection of enough statistics in a reasonable amount of computer time.

\footnotetext{
${ }^{1}$ Supported by Bundesministerium für Forschung und Technologie, Bonn, Germany.
}

In the present letter I describe an updating procedure with the fermion determinant evaluated to high order in the hopping parameter expansion at every link and included in the Monte Carlo updating. The use of the hopping parameter expansion of fermion determinant in the updating was already attempted previously in a different way by Lang and Nicolai [9] . They, however, sorted out the contributions of the fermion determinant to the effective action according to closed curves passing through a given link. The number of such curves increases very rapidly with the length (i.e. with the order of expansion): at 12 th order there are more than $4 \times 10^{6}$ such curves and e.g. at 16 th order already more than $6 \times 10^{9}[10]$. (Actually, these numbers refer to an infinite lattice. On a finite periodic lattice the number is still increased by the curves wrapping around the lattice.) It is clearly impossible to handle such large numbers of curves with any reasonable approximation (in ref. [9] typically 20 curves with length not more than 12 were taken). The solution I have chosen is to use the fast iterative method [11] for the evaluation of the hopping parameter expansion coefficients of the fermion determinant. This can be done up to some definite order in the quark part of effective gauge field action. The error due to the truncation of hopping parameter series can be controlled by running the iteration from time to time to higher order on some smaller sample set of links. Besides, the higher order part of the hopping parameter series, which is not included in the updating, can also be considered as a small correction and it can 
be taken without difficulty as a part of the expectation value following ref. [7]. In this way it is possible to keep the errors in the evaluation of fermion determinant under control. The numerical hopping parameter expansion method could be useful also in combination with the other methods [1-6], some of which can presumably achieve a higher speed in computation.

The contribution of virtual quark loops to the Monte Carlo updating of gauge fields is expressed by the quark determinants belonging to the new $\left(U^{\prime}\right)$ and old $(U)$ gauge field configuration:

$$
\begin{aligned}
& \exp \left(-\Delta S_{\mathrm{q}}^{\mathrm{eff}}\right) \equiv \operatorname{det} \frac{1-K M\left[U^{\prime}\right]}{1-K M[U]} \\
& \quad=\operatorname{det}\left(1-\frac{K\left(M\left[U^{\prime}\right]-M[U]\right)}{1-K M[U]}\right) \equiv \operatorname{det}(1-K D)
\end{aligned}
$$

Here $M[U]$ is the "hopping matrix" in the fermion part of the Wilson action [12],

$M_{x_{1} x_{2}}=\sum_{x, \mu}\left(1+\gamma_{\mu}\right) U(x, \mu) \delta_{x_{1}, x+\hat{\mu}} \delta_{x, x_{2}}$.

The notations are the usual ones: $x$ denotes lattice sites, $\mu$ is a (positive or negative) direction, $\hat{\mu}$ is the corresponding unit vector, $\gamma_{\mu}=-\gamma_{-\mu}$ is a hermitean Dirac-matrix and $U(x, \mu)$ is the gauge field variable on the link $x \rightarrow x+\hat{\mu} . K$ is the hopping parameter connected to the bare quark mass $m_{\mathrm{q}}$ (times lattice spacing $a$ ) by

$K=\left(8+2 a m_{\mathrm{q}}\right)^{-1}$.

In general, $K$ (and $m_{\mathrm{q}}$ ) is a diagonal matrix in quarkflavour space. In the numerical calculation I shall assume, for definiteness, that there are three (light) flavours with degenerate mass.

The determinant to be evaluated in eq. (1) is actually not a high-dimensional one, because $\Delta M \equiv M\left[U^{\prime}\right]$ $-M[U]$ has usually only a few non-zero elements. In principle it is possible to change the gauge configuration on several links simultaneously, but in what follows I shall only consider the conventional case when only a single link is changed. In this case we have

$$
\Delta M_{x_{1} x_{2}}=\sum_{x, \mu}\left(1+\gamma_{\mu}\right) \Delta U(x, \mu) \delta_{x_{1}, x+\hat{\mu}} \delta_{x, x_{2}} .
$$

This is non-zero only at the endpoints of the particular link, therefore the determinant to be evaluated has rank 24 (counting spin and colour, too).
If a systematic hopping parameter expansion of the change in the quark part of the effective action is required one can use, instead of eq. (1), the relation

$\Delta S_{\mathrm{q}}^{\mathrm{eff}}=\sum_{j=1}^{\infty} \frac{K^{j}}{j} \operatorname{Tr}\left(D^{j}\right)$,

where the matrix $D$ has the block form $D=\left(D_{i j}\right),(i, j$ $=1,2$ )

$$
\begin{aligned}
& D_{11}=\sum_{l=3}^{\infty} K^{l} M[U]_{x, x+\hat{\mu}}^{l}\left(1+\gamma_{\mu}\right) \Delta U(x, \mu), \\
& D_{12}=\sum_{l=4}^{\infty} K^{l} M[U]_{x, x}^{l}\left(1-\gamma_{\mu}\right) \Delta U(x, \mu)^{+}, \\
& D_{21}=\sum_{l=4}^{\infty} K^{l} M[U]_{x+\hat{\mu}, x+\hat{\mu}}^{l}\left(1+\gamma_{\mu}\right) \Delta U(x, \mu), \\
& D_{22}=\sum_{l=3}^{\infty} K^{l} M[U]_{x+\hat{\mu}, x}^{l}\left(1-\gamma_{\mu}\right) \Delta U(x, \mu)^{+} .
\end{aligned}
$$

Truncating the hopping parameter series in the determinant eq. (1) or in the expansion eq. (5) is, in principle, not equivalent. The numerical deviation in the actual calculation I have done (see later) is, however, rather small: typically less than $1 \%$ in the value of $\Delta S_{\mathfrak{q}}^{\text {eff }}$. Since in my computer code the determinant calculation was faster than the evaluation of the trace of powers of the matrix $D$ I used in most cases eq. (1).

To speed up the code for the evaluation of the required matrix elements of the quark propagator $(1-K M)^{-1}$, a useful observation is that it is enough to compute for half of the initial spin index values. In the Dirac-matrix representation, where $\gamma_{4}$ is given by

$\gamma_{4}=\operatorname{diag}(1,1,-1,-1)$,

this is trivial on links in direction 4 , since $\left(1 \pm \gamma_{4}\right)$ is non-zero only for half of the index values. For the other directions one can use, for instance $(k=1,2,3)$, $\left(1+\gamma_{k}\right) \frac{1}{2}\left(1-\gamma_{4}\right)=\left(1+\gamma_{k}\right) \frac{1}{2}\left(1+\gamma_{4}\right) \gamma_{k} \frac{1}{2}\left(1-\gamma_{4}\right)$.

This shows how the two lower components of e.g. $M[U]_{x, x+\hat{k}}^{l}\left(1+\gamma_{k}\right)$ can be expressed by the upper two.

The difficult task in the Monte Carlo calculation with unquenched Wilson fermions is the computation of matrix elements of the quark propagator 
$(1-K M)^{-1}$ needed in eq. (1) or (6). In order not to violate detailed balance in the updating this has to be done every time a link is changed in the gauge configuration. The fastest possibility to do this is to determine the hopping parameter expansion coefficients iteratively [11] and take the truncated series (or, if necessary, some of the Padé-approximants) as an approximation to the ratio of fermion determinants. After investing a substantial amount of effort to speed up this part of the code the computer program reached a performance where some first test calculations became possible on a modest size lattice. I have chosen a $6^{4}$ lattice at the SU(3) gauge coupling constant $\beta$ $=6 / g^{2}=5.70$. In order to have light quarks, the hopping parameter $K$ has to be chosen properly for this $\beta$ value. In the quenched meson mass calculations at $\beta$ $=5.70$ the pion mass (and hence, by definition, also the quark mass) vanishes at $K_{\mathrm{cr}}=0.1690[11,13]$. The effect of the quark determinant shifts $K_{\mathrm{cr}}$ to a somewhat lower value. On the basis of the estimate made in $\mathrm{SU}(2)$ with $N_{\mathrm{f}}=1$ quark flavours [8] a good guess seems to be $K=0.15$. (A qualitatively similar shift of the critical $K$ value was also observed in SU(3) in the previous low-order hopping parameter expansion calcultaion [14] .) For the number of light flavours $N_{\mathrm{f}}$ $=3$ was taken in the present calculation. The maximum order of the hopping parameter expansion for the quark determinant was $l_{\max }=16$. This is high enough at $\beta=5.70$ and $K=0.15$, because the last orders contribute only a few percent to the value of the determinant ratio. For the relative importance of the different orders see table 1 .

\section{Table 1}

The average relative magnitude of the different orders of hopping parameter expansion in the logarithm of the ratio of quark determinants (i.e. the difference of effective actions) occurring in the updating at $\beta=5.70, K=0.15$ on a $6^{4}$ lat tice with 10 Metropolis hits per link: Configuration A: for all offered changes; Configuration B: for the accepted changes.

\begin{tabular}{cll}
\hline Order & Configuration A & Configuration B \\
\hline 4 & $0.44 \pm 0.01$ & $0.37 \pm 0.02$ \\
6 & $0.28 \pm 0.01$ & $0.28 \pm 0.01$ \\
8 & $0.151 \pm 0.005$ & $0.167 \pm 0.008$ \\
10 & $0.074 \pm 0.004$ & $0.092 \pm 0.007$ \\
12 & $0.033 \pm 0.003$ & $0.048 \pm 0.008$ \\
14 & $0.015 \pm 0.001$ & $0.026 \pm 0.006$ \\
16 & $0.008 \pm 0.001$ & $0.018 \pm 0.004$ \\
\hline
\end{tabular}

An important property of the 16 th order series for the ratio of the quark determinants needed in the Metropolis updating procedure is that higher and lower order contributions are strongly correlated. This makes possible to estimate the result of the $l_{\max }=16$ order series from some lower order (e.g. $l_{\max }=8$ or $l_{\max }=12$ ) calculation. For instance, in the actual calculation at $\beta=5.70$ with 10 Metropolis hits per link the $l_{\max }=16$ result can be obtained in the average by multiplying the $l_{\max }=8$ number by an extrapolation factor $\lambda \cong 1.14$. The same factor needed from $l_{\max }$ $=12$ to $l_{\max }=16$ is $\lambda \cong 1.03$. This brings a substantial gain in computer time but, of course, increases the error for the fermion determinant. Monitoring the difference from time to time on several hundreds of links, it turned out that the estimates based on the extrapolation from $l_{\max }=8$ deviated in the average from the exact $l_{\max }=16$ value by $16 \%$. The corresponding average deviation for $l_{\max }=12$ extrapolated to $l_{\max }$ $=16 \mathrm{was} 5 \%$. The error in the determinant ratio caused by this extrapolation is far from being normally distributed. In most cases the deviation is much less than the average, but sometimes (in a few percent of cases) also errors in the order of $100 \%$ occur. It seems plausible that the effect of the few cases, where the error due to the extrapolation from the lower order to $l_{\max }=16$ is large, averages out and does not influence the updating process on the long run. It is also possible to improve the extrapolation to the higher orders by a more elaborate use of the covariance matrix among the lower and higher order expansion coefficients.

The Monte Carlo run on the $6^{4}$ lattices was started from a gauge configuration well equilibrated with the pure SU(3) gauge Wilson action at $\beta=5.70$. Then, as a warm-up, 20 sweeps with 10 Metropolis hits including the $\left(N_{\mathrm{f}}=3\right)$ quark determinant up to $l_{\max }=8$ order were performed. After this the configuration was duplicated and the updating with the $l_{\max }=16$ quarkdeterminant started on the two copies. At the beginning of every sweep 100-300 randomly chosen links were updated using the exact $l_{\max }=16$ order determinants. From this the extrapolation factor between $l_{\max }=8$ or $l_{\max }=12$ and $l_{\max }=16$ was determined and then the rest of the links were updated, in a randomly chosen order, either with $l_{\max }=8$ or $l_{\max }=12$ including the extrapolation factor to $l_{\max }=16$. Altogether 80 such sweeps were performed on both configurations. Always 10 Metropolis hits were done per 
Table 2

The expectation value of planar Wilson loops $W(I, J)=\operatorname{Tr}(\ldots) / 3$ and the corresponding $\chi$-ratios $\chi(I, J)=-\ln \{[W(I, J) W(I-1, J-1)] /$ $\{W(I-1, J) W(I, J-1)]\}$ for $\beta=5.70, N_{\mathrm{f}}=0$ and for the two configurations A and B with $\beta=5.70, N_{\mathrm{f}}=3, K=0.15$. The errors for $N_{\mathrm{f}}=0$ were determined by making 6 groups out of the 120 measurements. The errors given for columns $A$ and B were obtained by considering the 30 measurements as independent.

\begin{tabular}{|c|c|c|c|}
\hline & \multirow[t]{2}{*}{$N_{\mathrm{f}}=0$} & \multicolumn{2}{|l|}{$N_{\mathrm{f}}=3$} \\
\hline & & Configuration A & Configuration B \\
\hline$w(1,1)$ & $0.5472 \pm 0.0015$ & $0.5754 \pm 0.0012$ & $0.5720 \pm 0.0013$ \\
\hline$W(1,2)$ & $0.3223 \pm 0.0020$ & $0.3589 \pm 0.0014$ & $0.3544 \pm 0.0015$ \\
\hline$w(2,2)$ & $0.1299 \pm 0.0019$ & $0.1621 \pm 0.0012$ & $0.1585 \pm 0.0011$ \\
\hline$W(1,3)$ & $0.1928 \pm 0.0019$ & $0.2290 \pm 0.0016$ & $0.2234 \pm 0.0017$ \\
\hline$w(2,3)$ & $0.0561 \pm 0.0016$ & $0.0794 \pm 0.0012$ & $0.0755 \pm 0.0010$ \\
\hline$W(3,3)$ & $0.0194 \pm 0.0010$ & $0.0340 \pm 0.0010$ & $0.0294 \pm 0.0009$ \\
\hline$\chi(2,2)$ & $0.379 \pm 0.015$ & $0.323 \pm 0.017$ & $0.326 \pm 0.018$ \\
\hline$x(2,3)$ & $0.326 \pm 0.028$ & $0.264 \pm 0.033$ & $0.280 \pm 0.032$ \\
\hline$x(3,3)$ & $0.222 \pm 0.045$ & $0.134 \pm 0.066$ & $0.202 \pm 0.064$ \\
\hline
\end{tabular}

link. For the quark fields periodic boundary conditions were assumed (similarly to the gauge fields). The updating time for a single link with $l_{\max }=8$ was $2 \mathrm{~s}$ on the Siemens 7.882 (or IBM 3081D). The $l_{\max }=12$ updating is about 8 times slower. Roughly $2 / 3$ of the time was spent with $l_{\max }=8$ and $1 / 3$ with $l_{\max }=12$, both extrapolated to $l_{\max }=16$. The average extrapolation factors were, respectively, $\lambda=1.14 \pm 0.02$ and $\lambda=1.03 \pm 0.01$. After each of the last 30 sweeps the rectangular Wilson loop expectation values were determined on both configurations. The results are collected in table 2, where, for comparison, pure gauge results at $\beta=5.70$ on a $6^{4}$ lat tice are also given. These latter were obtained in 120 measurements separated by 4 sweeps. The qualitative difference between the pure gauge and unquenched Wilson-loop values corresponds to the expectation: the single plaquette value, for instance, is increased by more than $4 \%$ and the larger Wilson loops in the table show an increase up to $50 \%$, consistent with screening of the colour charge. The picture is qualitatively similar to the $N_{\mathrm{f}}=1$ results obtained in ref. [7] with SU(2) gauge field but the screening is much more evident. The statistics (and the lattice itself) is not large enough for drawing definite quantitative conclusions concerning the $\chi$-ratios [15] or other related quantities relevant for the quark-antiquark potential. This will be attempted in a subsequent run on $8^{4}$ lattice.

The purpose of this letter was to demonstrate that an unquenched updating of some reasonably sized lattices is feasible for Wilson fermions by the use of the numerical hopping parameter expansion technique. The code can certainly be made faster even on a serial computer, but a substantial gain in performance is to be expected on a vector machine like the CYBER 205. In fact, the important part of the code (namely, the iteration for the hopping parameter expansion coefficients) is well suited for vectorization. The method is practicable also for larger lattices and for higher orders in the hopping parameter expansion (needed for larger $\beta$ 's). For a given order in hopping parameter expansion (and large lattice volume) the required CPU time grows linearly with the lattice volume. Similarly, for a given lattice volume (and large enough order) the necessary CPU time grows also linearly with the order. An advantage of the hopping parameter expansion method is its flexibility and the possibility of a good control of errors.

I wish to thank the Computer Center of the University of Hamburg for the generous support of this computation on the Siemens 7.882 .

\section{References}

[1] F. Fucito, E. Marinari, G. Parisi and C. Rebbi, Nucl. Phys. B1 80 [FS3] (1981) 369; H.W. Hamber, E. Marinari, G. Parisi and C. Rebbi, Phys. Lett. 124B (1983) 99; SACLAY preprint SPh. T/83/54 (1983). 
[2] G. Bhanot, U.M. Heller and I.O. Stamatescu, Phys. Lett. 129B (1983) 440.

[3] S. Otto and M. Randeira, Nucl. Phys. B220 (1983) 479; S. Otto, CALTECH preprint CALT-68-1041 (1983).

[4] J. Kuti, Phys. Rev. Lett. 49 (1982) 183; in: Proc. Johns Hopkins Workshop (Florence, 1982).

[5] B. Berg and D. Foerster, Phys. Lett. 106B (1981) 323.

[6] J. Polonyi and H.M. Wyld, Phys. Rev. Lett. 51 (1983) 2257.

[7] H. Joos and I. Montvay, Nucl. Phys. B225 [FS9] (1983) 565.

[8] I. Montvay, Phys. Lett. 132B (1983) 393.

[9] C.B. Lang and H. Nicolai, Nucl. Phys. B200 [FS4] (1982) 135 .
[10] B. Berg, A. Billoire and D. Foerster, Lett. Math. Phys. 6 (1982) 293.

[11] P. Hasenfratz and I. Montvay, Phys. Rev. Lett. 50 (1983) 309; DESY preprint 83-072 (1983), to be published in Nucl. Phys. B.

[12] K.G. Wilson, Phys. Rev. D10 (1974) 2445.

[13] K.C. Bowler, G.S. Pawley and D.J. Wallace, in: Proc. Intern. Europhysics Conf. on High energy physics (Brighton, 1983).

[14] A. Hasenfratz, P. Hasenfratz, Z. Kunszt and C.B. Lang, Phys. Lett. 110B (1982) 282.

[15] M. Creutz, Phys. Rev. D21 (1980) 2308. 